\title{
Isometric immersions of warped products
}

\author{
M. Dajczer and T. Vlachos
}

\begin{abstract}
We provide conditions under which an isometric immersion of a (warped) product of manifolds into a space form must be a (warped) product of isometric immersions.
\end{abstract}

A basic problem in the theory of submanifolds is to provide conditions that imply that an isometric immersion of a product manifold must be a product of isometric immersions. The first contribution to that problem was given by Moore [7] under purely intrinsic assumptions. Namely, he showed that an isometric immersion of a product of Riemannian manifolds

$$
f: M^{n}=M_{1}^{m_{1}} \times \ldots \times M_{k}^{m_{k}} \rightarrow \mathbb{R}^{n+k}
$$

must be a product $f=f_{1} \times \cdots \times f_{k}$ of hypersurfaces $f_{i}: M_{i}^{m_{i}} \rightarrow \mathbb{R}^{m_{i}+1}$ if the codimension equals the number of factors and no factor has an open subset at which all sectional curvatures vanish.

Moore's local result trivially fails if higher codimension is allowed. For instance, one can compose $f$ as above with any (local) isometric immersion of $\mathbb{R}^{n+k}$ into $\mathbb{R}^{n+k+\ell}$ for $\ell \geq 1$. This suggest that for higher codimension a hypothesis of extrinsic nature that forbids compositions may be needed. In that direction, a natural assumption is an upper bound on the size of the $s$-nullities of the immersion; see Example 7 below. The concept of $s$-nullities was introduced in [3] and has been playing an increasing role in the study of rigidity questions of submanifolds; see [3], [4] and [6].

In this paper, we provide conditions under which an isometric immersion of a product of manifolds must be a product of immersions. In fact, we consider a much more general situation. We allow the ambient space to have any constant sectional curvature and the submanifold can be a warped product of manifolds instead of just an ordinary Riemannian product. 
We point out that the very special case of a warped product of only two factors in codimension two was solved in [5] in great generality, namely, without any assumption of extrinsic nature.

\section{Preliminaries}

On a product $M^{n}=M_{0} \times M_{1} \times \ldots \times M_{k}$ of connected Riemannian manifolds we define a new metric by

$$
\langle X, Y\rangle=\left\langle\pi_{0 *} X, \pi_{0 *} Y\right\rangle+\sum_{i=1}^{k}\left(\rho_{i} \circ \pi_{0}\right)^{2}\left\langle\pi_{i *} X, \pi_{i *} Y\right\rangle
$$

where $\rho_{1}, \ldots, \rho_{k} \in C^{\infty}\left(M_{0}\right)$ are positive functions and $\pi_{i}: M^{n} \rightarrow M_{i}$ denotes the canonical projection. We call $M^{n}$ endowed with this metric the warped product of $M_{0}, \ldots, M_{k}$ with warping functions $\rho_{1}, \ldots, \rho_{k}$ and denote by

$$
M^{n}=M_{0} \times_{\rho_{1}} M_{1} \times \ldots \times{ }_{\rho_{k}} M_{k} .
$$

For simplicity, from now on we identify the tangent bundles $T M_{j}$ with the corresponding tangent distributions to $M^{n}$.

Let $\mathbb{Q}_{c}^{m}$ denote a complete and simply connected space form of sectional curvature $c$. If $c \neq 0$, we always view $\mathbb{Q}_{c}^{m}$ as a totally umbilical hypersurface of Euclidean space $\mathbb{R}^{m+1}$ with Riemannian or Lorentzian signature according to the sign of $c$. Fix a point $q \in \mathbb{Q}_{c}^{m}$ and let $\mathbb{Q}_{c}^{m_{0}}, \mathbb{Q}_{c_{1}}^{m_{1}}, \ldots, \mathbb{Q}_{c_{k}}^{m_{k}}, m=\sum m_{j}$, be submanifolds through $q$ such that the first one is totally geodesic and all the others are totally umbilical with mean curvature vectors $z_{1}, \ldots, z_{k}$ at $q$ and $\left\langle z_{i}, z_{j}\right\rangle=-c$ for $i \neq j$. The warped product representation

$$
\Psi: \mathbb{Q}_{c}^{m_{0}} \times_{\sigma_{1}} \mathbb{Q}_{c_{1}}^{m_{1}} \times \ldots \times_{\sigma_{k}} \mathbb{Q}_{c_{k}}^{m_{k}} \rightarrow \mathbb{Q}_{c}^{m}
$$

of $\mathbb{Q}_{c}^{m}$ is the map

$$
\Psi\left(p_{0}, p_{1}, \ldots, p_{k}\right)=p_{0}+\sum_{i=1}^{k} \sigma_{i}\left(p_{0}\right)\left(p_{i}-q\right)
$$

where the functions $\sigma_{i}: \mathbb{Q}_{c}^{m_{0}} \rightarrow \mathbb{R}_{+}$are defined as

$$
\sigma_{i}(p)= \begin{cases}1+\left\langle p-q, a_{i}\right\rangle & \text { if } c=0 \\ \left\langle p, a_{i}\right\rangle & \text { if } c \neq 0\end{cases}
$$

and satisfy $\sigma_{i}(q)=1$ with $a_{i}=c q-z_{i}$. 
It was shown by Nölker [8] that any isometry of a warped product with $k+1$ factors onto an open dense subset of $\mathbb{Q}_{c}^{m}$ arises as the restriction of a warped product representation as above.

Given a warped product representation

$$
\Psi: \mathbb{Q}_{c}^{m_{0}} \times_{\sigma_{1}} \mathbb{Q}_{c_{1}}^{m_{1}} \times \ldots \times_{\sigma_{k}} \mathbb{Q}_{c_{k}}^{m_{k}} \rightarrow \mathbb{Q}_{c}^{n+p}
$$

and isometric immersions $f_{i}: M_{i} \rightarrow \mathbb{Q}_{c_{i}}^{m_{i}}, 0 \leq i \leq k$ with $c_{0}=c$, the map

$$
f=\Psi \circ\left(f_{0} \times \ldots \times f_{k}\right): M^{n}=M_{0} \times_{\rho_{1}} M_{1} \times \ldots \times{ }_{\rho_{k}} M_{k} \rightarrow \mathbb{Q}_{c}^{n+p}
$$

is an isometric immersion of the warped product manifold $M^{n}$ with warping functions $\rho_{i}=\sigma_{i} \circ f_{0}$.

We call $f$ given by (21) a warped product of isometric immersions. It is easy to see that its second fundamental form

$$
\alpha: T M \times T M \rightarrow N_{f} M
$$

is adapted to the product structure of $M^{n}$. This means that

$$
\alpha\left(X_{i}, X_{j}\right)=0 \text { for all } X_{i} \in T M_{i}, X_{j} \in T M_{j}, \quad i \neq j .
$$

The following basic result is due to Nölker [8].

Proposition 1. Let $f: M^{n}=M_{0} \times_{\rho_{1}} M_{1} \times \ldots \times{ }_{\rho_{k}} M_{k} \rightarrow \mathbb{Q}_{c}^{n+p}$ be an isometric immersion with adapted second fundamental form. Then, there is a warped product representation $\Psi$ of $\mathbb{Q}_{c}^{n+p}$ and isometric immersions $f_{i}: M_{i} \rightarrow \mathbb{Q}_{c_{i}}^{m_{i}}$, $0 \leq i \leq k$, such that $f=\Psi \circ\left(f_{0} \times \ldots \times f_{k}\right)$ is a warped product of isometric immersions.

We now consider isometric immersions of a Riemannian product, i.e., all warping functions are constant. The following fact was used by Moore to prove the result discussed in the introduction.

Corollary 2. Let $f: M^{n}=M_{1} \times \ldots \times M_{k} \rightarrow \mathbb{R}^{n+p}$ be an isometric immersion with adapted second fundamental form. Then $f=f_{1} \times \ldots \times f_{k}$ is a product of isometric immersions $f_{i}: M_{i} \rightarrow \mathbb{R}^{n_{i}}, 1 \leq i \leq k$. 
For the case $c \neq 0$, we first observe that by fixing a point $\bar{p} \in \mathbb{Q}_{c}^{m_{0}}$ in the warped product representation (1) we obtain the isometric embedding $F: \mathbb{Q}_{c_{1}}^{m_{1}} \times \ldots \times \mathbb{Q}_{c_{k}}^{m_{k}} \rightarrow \mathbb{Q}_{c}^{n+p}$ with parallel second fundamental form and flat normal bundle given by

$$
F\left(p_{1}, \ldots, p_{k}\right)=\Psi\left(\bar{p}, p_{1}, \ldots, p_{k}\right) .
$$

The following result is due to Reckziegel [9].

Proposition 3. Let $f: M^{n}=M_{1} \times \ldots \times M_{k} \rightarrow \mathbb{Q}_{c}^{n+p}, c \neq 0$, be an isometric immersion with adapted second fundamental form. Then, there are isometric immersions $f_{i}: M_{i} \rightarrow \mathbb{Q}_{c_{i}}^{m_{i}}, 1 \leq i \leq k$, such that

$$
f=F \circ\left(f_{1} \times \cdots \times f_{k}\right)
$$

where $F$ is given by (3).

\section{The main lemma}

Let $\beta: V \times V \rightarrow W$ be a symmetric bilinear form where $V$ and $(W,\langle\rangle$,$) are$ real vector spaces of finite dimension $n$ and $p$, respectively, equipped with inner products.

The $s$-nullity $\nu_{s}$ of $\beta$ for any integer $1 \leq s \leq p$ is defined by

$$
\nu_{s}=\max _{U^{s} \subset W} \operatorname{dim}\left\{x \in V: \beta_{U^{s}}(x, y)=0 \text { for all } y \in V\right\} .
$$

Here $\beta_{U^{s}}=\pi_{U^{s}} \circ \beta$ where $U^{s}$ is any $s$-dimensional subspace of $W$ and $\pi_{U^{s}}: W \rightarrow U^{s}$ denotes the orthogonal projection.

Let $R: V \times V \times V \times V \rightarrow \mathbb{R}$ be the multilinear map with the algebraic properties of the curvature tensor defined by

$$
R(x, y, z, w)=\langle\beta(x, w), \beta(y, z)\rangle-\langle\beta(x, z), \beta(y, w)\rangle .
$$

Lemma 4. Assume that $2 p<n$ and $\nu_{s}<n-2 s$ for all $1 \leq s \leq p$. Let $V=V_{1} \oplus V_{2}$ be an orthogonal splitting such that

$$
R(x, y, z, u)=R(x, y, u, v)=R(x, u, v, w)=0
$$

for any $x, y, z \in V_{1}$ and $u, v, w \in V_{2}$. Then,

$$
S=\operatorname{span}\left\{\beta(x, y): x \in V_{1} \text { and } y \in V_{2}\right\}=0 \text {. }
$$


Proof: For $x \in V_{1}$ we denote by $B_{x}: V_{2} \rightarrow S$ the linear map

$$
B_{x}(y)=\beta(x, y)
$$

and set

$$
D=\operatorname{ker} B_{x} \subset V_{2} .
$$

Fix $x \in V_{1}$ such that $B_{x}$ has maximal rank, i.e.,

$$
\operatorname{rank} B_{x} \geq \operatorname{rank} B_{y}
$$

for any $y \in V_{1}$. Thus, $\operatorname{dim} D \leq \operatorname{dim} \operatorname{ker} B_{y}$ for any $y \in V_{1}$.

We first argue that

$$
D \subset \operatorname{ker} B_{y} \text { for any } y \in V_{1} \text {. }
$$

From $R\left(x, y, v, e_{j}\right)=0$ we obtain

$$
\left\langle B_{x} e_{j}, B_{y} v\right\rangle=\left\langle B_{x} v, B_{y} e_{j}\right\rangle=0, \quad 1 \leq j \leq \ell,
$$

where $v \in D, y \in V_{1}$ and $\left\{e_{1}, \ldots, e_{\ell}\right\}$ is an orthonormal basis of $E$ in the orthogonal splitting $V_{2}=D \oplus E$.

The rank of $B_{x+t y}$ is at most $\ell$ for any $t \in \mathbb{R}$. Therefore, the vectors $B_{x+t y} v=t B_{y} v, B_{x+t y} e_{j}, 1 \leq j \leq \ell$, are linearly dependent. Hence, the Gramm determinant of these vectors is an identically zero polynomial in $t$. By (17) the term of lowest order is $t^{2}\left\|B_{y} v\right\|^{2} G$, where $G$ is the Gramm determinant of the linearly independent vectors $B_{x} e_{j}, 1 \leq j \leq \ell$. It follows that $B_{y} v=0$ for any $y \in V_{1}$ and $v \in D$, and this is (6).

Next, we prove that

$$
\beta(u, v)-\sum_{i, j=1}^{\ell}\left\langle u, e_{i}\right\rangle\left\langle v, e_{j}\right\rangle \beta\left(e_{i}, e_{j}\right) \in S^{\perp}
$$

for any $u, v \in V_{2}$. From ([6]) we obtain

$$
\beta(y, v)=\sum_{j=1}^{\ell}\left\langle v, e_{j}\right\rangle \beta\left(y, e_{j}\right)
$$

for any $y \in V_{1}$. Then $R(y, u, w, v)=0$ yields

$$
\langle\beta(u, v), \beta(y, w)\rangle=\sum_{j=1}^{\ell}\left\langle v, e_{j}\right\rangle\left\langle\beta\left(y, e_{j}\right), \beta(u, w)\right\rangle
$$


for any $y \in V_{1}$ and $u, v, w \in V_{2}$. In particular,

$$
\left\langle\beta(w, u), \beta\left(y, e_{j}\right)\right\rangle=\sum_{i=1}^{\ell}\left\langle u, e_{i}\right\rangle\left\langle\beta\left(y, e_{i}\right), \beta\left(e_{j}, w\right)\right\rangle .
$$

Hence,

$$
\langle\beta(u, v), \beta(y, w)\rangle=\sum_{i, j=1}^{\ell}\left\langle u, e_{i}\right\rangle\left\langle v, e_{j}\right\rangle\left\langle\beta\left(y, e_{i}\right), \beta\left(e_{j}, w\right)\right\rangle .
$$

Using

$$
\left\langle\beta\left(y, e_{i}\right), \beta\left(e_{j}, w\right)\right\rangle=\left\langle\beta(y, w), \beta\left(e_{i}, e_{j}\right)\right\rangle
$$

we obtain

$$
\left\langle\beta(u, v)-\sum_{i, j=1}^{\ell}\left\langle u, e_{i}\right\rangle\left\langle v, e_{j}\right\rangle \beta\left(e_{i}, e_{j}\right), \beta(y, w)\right\rangle=0
$$

for any $y \in V_{1}$ and $u, v, w \in V_{2}$, and this is (8).

We have from (6) that $\beta(u, y)=0$ if $u \in D$ and $y \in V_{1}$ and from (8) that $\beta_{S}(u, v)=0$ if $u \in D$ and $v \in V_{2}$. Therefore,

$$
\beta_{S}(u, e)=0 \text { if } u \in D \text { and } e \in V \text {. }
$$

Suppose that $s=\operatorname{dim} S \neq 0$. Then, choose vectors $x_{j} \in V_{j}, j=1,2$, such that $B_{x_{j}}: V_{k} \rightarrow S, j \neq k$, has maximal rank. It follows from the above that $\beta_{S}(b, e)=0$ for any $b \in \operatorname{ker} B_{x_{1}} \oplus \operatorname{ker} B_{x_{2}}$ and $e \in V$. Hence,

$$
\nu_{s} \geq \operatorname{dim} \operatorname{ker} B_{x_{1}}+\operatorname{dim} \operatorname{ker} B_{x_{2}} \geq n-2 s,
$$

and this contradicts our assumption.

\section{The results}

In this section, we state and prove the results of this paper. The main tool for the proofs is the algebraic lemma given in the preceding section.

We define the s-nullity $\nu_{s}(x)$ of an isometric immersion $f: M^{n} \rightarrow \tilde{M}^{n+p}$ at a point $x \in M^{n}$ for an integer $1 \leq s \leq p$ as the $s$-nullity of its second 
fundamental form at that point. Notice that $\nu_{p}(x)$ is the standard index of relative nullity of $f$ at $x \in M^{n}$.

We start with the case of a Riemannian products of manifolds

$$
M^{n}=M_{1}^{n_{1}} \times \ldots \times M_{k}^{n_{k}} .
$$

Theorem 5. Let $f: M^{n} \rightarrow \mathbb{Q}_{c}^{n+p}$ with $2 p<n$ be an isometric immersion such that $\nu_{s}<n-2 s$ for $1 \leq s \leq p$ at any point.

(i) If $c=0$, then $f=f_{1} \times \ldots \times f_{k}$ is a product of isometric immersions.

(ii) If $c \neq 0$, then $f=F \circ\left(f_{1} \times \cdots \times f_{k}\right)$ where $F$ is given by (3).

Proof: We apply Lemma 4 to the second fundamental form of $f$ at any point of $M^{n}$, and conclude that it must be adopted to the product structure of the manifold. The proof now follows from Corollary 2 or Proposition 3 according to $c=0$ or $c \neq 0$.

In the remaining of the paper we consider the case of isometric immersions of warped product manifolds

$$
M^{n}=M_{0}^{n_{0}} \times_{\rho_{1}} M_{1}^{n_{1}} \times \ldots \times_{\rho_{k}} M_{k}^{n_{k}} .
$$

Our next result assumes that the warping functions are pairwise linearly independent. This condition should not be seen as a restriction. In fact, if two warping functions are linearly dependent, we may change the metric of one of the factors by an homothety in such a way that both factors now have the same warping function and can be put together in a new factor.

Before we give the statements for the warped product case, we recall the relations between the Levi-Civita connections and the curvature tensors for a warped product metric (left hand side) and the Riemannian product metric:

$$
\nabla_{X} Y=\tilde{\nabla}_{X} Y+\sum_{j=1}^{k}\left(\left\langle X^{j}, Y^{j}\right\rangle \eta_{j}-\left\langle X, \eta_{j}\right\rangle Y^{j}-\left\langle Y, \eta_{j}\right\rangle X^{j}\right)
$$

and

$$
\begin{aligned}
R(X, Y) & =\tilde{R}(X, Y)-\sum_{i, j=1}^{k}\left\langle\eta_{i}, \eta_{j}\right\rangle X^{i} \wedge Y^{j} \\
& +\sum_{j=1}^{k}\left[\left(\nabla_{X^{0}} \eta_{j}-\left\langle\eta_{j}, X\right\rangle \eta_{j}\right) \wedge Y^{j}+X^{j} \wedge\left(\nabla_{Y^{0}} \eta_{j}-\left\langle\eta_{j}, Y\right\rangle \eta_{j}\right)\right]
\end{aligned}
$$


where $X^{j}=\left(\pi_{j}\right)_{*} X$ and $\eta_{j}=-\operatorname{grad} \log \rho_{j}$.

Theorem 6. Let $f: M^{n} \rightarrow \mathbb{Q}_{c}^{n+p}$ with $2 p<n$ be an isometric immersion such that $\nu_{s}<n-2 s$ for $1 \leq s \leq p$ at any point. Assume that the warping functions are pairwise linearly independent everywhere. Then $f$ is a warped product of isometric immersions.

Proof: We have from (10) that

$$
R(X, Y, Z, U)=R(X, Y, U, V)=R(X, U, V, W)=0
$$

for any $X, Y, Z \in T M_{0}$ and $U, V, W \in T M_{1} \oplus \cdots \oplus T M_{k}$. Hence Lemma 4 applies to the second fundamental form of $f$ at any point of $M^{n}$. Thus,

$$
\alpha(X, V)=0
$$

if $X \in T M_{0}$ and $V \in T M_{1} \oplus \cdots \oplus T M_{k}$.

To conclude that the second fundamental form of the immersion must be adopted to the product structure, it remains to show that

$$
\alpha(U, V)=0 \text { if } U \in T M_{i} \text { and } V \in T M_{j} \text { for } i \neq j \text { and } i, j \geq 1 .
$$

To see this, first observe that (91) gives

$$
\nabla_{U} V=0 \text { and } \nabla_{X} U=\nabla_{U} X=-\left\langle\eta_{i}, X\right\rangle U
$$

for any $X \in T M_{0}$. The Codazzi equation

$$
\left(\nabla_{X}^{\perp} \alpha\right)(U, V)=\left(\nabla_{U}^{\perp} \alpha\right)(X, V)
$$

of $f$ using (11) and (13) yields

$$
\left(\nabla_{X}^{\perp} \alpha\right)(U, V)=\alpha\left(\nabla_{X} U, V\right) .
$$

Since the left hand side is symmetric in $U$ and $V$, it follows using (13) that

$$
\alpha(U, V)\left\langle\eta_{i}-\eta_{j}, X\right\rangle=0
$$

for any $X \in T M_{0}$. Thus (12) holds since $\eta_{i}-\eta_{j} \neq 0$ if $i \neq j$ by assumption, and the proof follows from Proposition 1 . 
Example 7. The assumption on the $s$-nullities in Theorem 6 goes beyond excluding the case of compositions as discussed in the introduction. In fact, for submanifolds with two factors in codimension two it was shown in [5] that there are two families of submanifolds that are not warped products of isometric immersions. Although the submanifolds belonging to one family are compositions, the ones in the other family are not.

As in Moore's result discussed in the introduction, we next restrict the codimension to the number of factors and assume a curvature condition.

Theorem 8. Let $f: M^{n}=M_{0}^{n_{0}} \times_{\rho_{1}} M_{1}^{n_{1}} \times \ldots \times{ }_{\rho_{k}} M_{k}^{n_{k}} \rightarrow \mathbb{Q}_{c}^{n+k+1}$ with $2 k+2<n$ be an isometric immersion such that $\nu_{s}<n-2 s$ for $1 \leq s \leq k+1$ at any point. Assume that no factor has an open subset where the sectional curvature or the corresponding warping function is constant. Then $f$ is a warped product of hypersurfaces.

Proof: If the number $r$ of pairwise linearly independent warping functions is $r=k$, the result follows from Theorem 6 and the curvature assumption. Thus, we may assume $r<k$ and let $\rho_{i_{1}}, \ldots, \rho_{i_{r}}, 1 \leq i_{1}<\ldots<i_{r} \leq k$, be the pairwise linearly independent warping functions. Hence, we may view $M^{n}$ as a warped product

$$
M^{n}=M_{0}^{\ell_{0}} \times_{\rho_{i_{1}}} \hat{M}_{1}^{\ell_{1}} \times \cdots \times_{\rho_{i_{r}}} \hat{M}_{r}^{\ell_{r}}
$$

where the factors $\hat{M}_{j}^{\ell_{j}}$ are the Riemannian products

$$
\hat{M}_{j}^{\ell_{j}}=\Pi_{i \in I_{j}} M_{i}, 1 \leq j \leq r,
$$

and $I_{j}$ denotes the set of all indices $1 \leq i \leq k$ that correspond to factors with the same associated warping function after homoteties, if necessary.

We apply Theorem 6 to $M^{n}$ with the warped product structure (14). It follows that $f$ is a warped product of isometric immersions

$$
f=\Psi \circ\left(\hat{f}_{0} \times \hat{f}_{1} \times \cdots \times \hat{f}_{r}\right)
$$

with respect to a warped product representation

$$
\Psi: \mathbb{Q}_{c}^{m_{0}} \times_{\sigma_{1}} \mathbb{Q}_{c_{1}}^{m_{1}} \times \cdots \times_{\sigma_{r}} \mathbb{Q}_{c_{r}}^{m_{r}} \rightarrow \mathbb{Q}_{c}^{n+k+1},
$$

where $\hat{f}_{0}=f_{0}$ and $\hat{f}_{j}: \hat{M}_{j}^{\ell_{j}} \rightarrow \mathbb{Q}_{c_{j}}^{m_{j}}, \quad 1 \leq j \leq r$. 
We show that $\hat{f}_{j}, 1 \leq j \leq r$, satisfies at any point of $\hat{M}_{j}^{\ell_{j}}$ that

$$
\nu_{s}^{\hat{f}_{j}}<\ell_{j}-2 s
$$

for $1 \leq s \leq \operatorname{cod}\left(\hat{f}_{j}\right)=m_{j}-\ell_{j}$. We have from Lemma 12 in [8] that

$$
\alpha=\Psi_{*} \alpha^{\hat{f}_{0} \times \cdots \times \hat{f}_{r}},
$$

where

$$
\alpha^{\hat{f}_{0} \times \cdots \times \hat{f}_{r}}(X, Y)=\left(\gamma(X, Y), \alpha^{\hat{f}_{1}}\left(\hat{X}_{1}, \hat{Y}_{1}\right), \ldots, \alpha^{\hat{f}_{r}}\left(\hat{X}_{r}, \hat{Y}_{r}\right)\right)
$$

and

$$
\gamma(X, Y)=\alpha^{\hat{f}_{0}}\left(X_{0}, Y_{0}\right)-\sum_{j=1}^{r} \rho_{i_{j}}\left\langle\hat{X}_{j}, \hat{Y}_{j}\right\rangle\left(\operatorname{grad} \sigma_{j}-f_{0 *} \operatorname{grad} \rho_{i_{j}}\right) .
$$

We argue by contradiction. At some point of $\hat{M}_{j_{0}}^{\ell_{0}}$, let $\nu_{s_{0}}^{\hat{f}_{j_{0}}} \geq \ell_{j_{0}}-2 s_{0}$ for some $1 \leq j_{0} \leq r$ and $1 \leq s_{0} \leq \operatorname{cod}\left(\hat{f}_{j_{0}}\right)$. Therefore, there exists a subspace $U^{s_{0}} \subset N_{\hat{f}_{j_{0}}} \hat{M}_{j_{0}}$ such that

$$
\operatorname{dim}\left\{Y \in T M_{j_{0}}: \alpha_{U^{s_{0}}}^{\hat{f}_{j_{0}}}(Y, Z)=0 \text { for all } Z \in T M_{j_{0}}\right\} \geq \ell_{j_{0}}-2 s_{0} .
$$

From the above, we have

$$
\alpha_{U^{s_{0}}}^{\hat{f}_{0} \cdots \times \hat{f}_{r}}(X, Y)=\left(0,0, \ldots, \alpha_{U^{s_{0}}}^{\hat{f}_{j_{0}}}\left(\hat{X}_{j_{0}}, \hat{Y}_{j_{0}}\right), \ldots, 0\right) .
$$

It follows that

$$
\nu_{s_{0}}\left(\hat{f}_{0} \times \cdots \times \hat{f}_{r}\right) \geq l_{j_{0}}-2 s_{0}+\sum_{i \neq j_{0}} l_{i}=n-2 s_{0},
$$

In view of (16) this a contradiction and proves (15).

Assume that $\left|I_{j}\right|>1$ for some $j$. From (15) and Theorem [5, we obtain

$$
\hat{f}_{j}= \begin{cases}g_{1}^{j} \times \cdots \times g_{\left|I_{j}\right|}^{j} & \text { if } c_{j}=0, \\ F_{j} \circ\left(g_{1}^{j} \times \cdots \times g_{\left|I_{j}\right|}^{j}\right) & \text { if } c_{j} \neq 0 .\end{cases}
$$


By the curvature assumption $\operatorname{cod}\left(g_{i}^{j}\right) \geq 1$ for any $i \in I_{j}$. Therefore,

$$
\operatorname{cod}\left(\hat{f}_{j}\right) \geq\left|I_{j}\right| \text { if } c_{j}=0 \text { and } \operatorname{cod}\left(\hat{f}_{j}\right)>\left|I_{j}\right| \text { if } c_{j} \neq 0
$$

We also have from the curvature assumption that $\operatorname{cod}\left(\hat{f}_{j}\right) \geq 1$ if either $j=0$ or $\left|I_{j}\right|=1$. Hence,

$$
k+1=\sum_{j=0}^{r} \operatorname{cod}\left(\hat{f}_{j}\right) \geq \sum_{j=0}^{r}\left|I_{j}\right|=k+1 .
$$

Therefore $\operatorname{cod}\left(\hat{f}_{j}\right)=\left|I_{j}\right|$ for any $0 \leq j \leq r$. In particular, if $\left|I_{j}\right|>1$ then $\hat{f}_{j}$ is a product of Euclidean hypersurfaces. We conclude that each factor in the initial product decomposition of $M^{n}$ must be a hypersurface.

Remark 9. Notice that the proof of the last result gives some additional information. For instance, if the warping functions are not pairwise linearly independent then we must have $c \leq 0$.

The results of this paper are local in nature. Global results for isometric immersions of Riemannian products where obtained in [1] and [2]. In the latter the role of compositions was clarified.

\section{References}

[1] S. Alexander and R. Maltz, Isometric immersions of Riemannian products in Euclidean space. J. Differential Geom. 11 (1976), 47-57.

[2] J. Barbosa, M. Dajczer and R. Tojeiro, Isometric immersions of Riemannian products revisited. Comment. Math. Helv. 69 (1994), 281-290.

[3] M. do Carmo and M. Dajczer, Conformal Rigidity. Amer. J. Math. 109 (1987), 963-985.

[4] M. Dajczer and L. Florit, Genuine deformations of submanifolds. Comm. Anal. Geom. 12 (2004), 1105-1121.

[5] M. Dajczer and R. Tojeiro, Isometric immersions in codimension two of warped products into space forms. Illinois J. Math. 48 (2004), 711-746. 
[6] M. Dajczer and R. Tojeiro, Submanifolds with nonparallel first normal bundle revisited. Preprint.

[7] J. D. Moore, Isometric immersions of Riemannian products. J. Differential Geom. 5 (1971), 159-168.

[8] S. Nölker, Isometric immersions of warped products. Differential Geom. Appl. 6 (1996), 1-30.

[9] H. Reckziegel, Hypersurfaces with parallel Ricci tensor in spaces of constant curvature. Results in Math. 27 (1995), 113-116.

IMPA - Estrada Dona Castorina, 110 22460-320 - Rio de Janeiro - Brazil E-mail: marcos@impa.br
Univ. of Ioannina - Math. Dept. 45110 Ioannina - Greece E-mail: tvlachos@uoi.gr 